\title{
VERÄNDERUNGEN IN DER NORWEGISCHEN SAAMENPOLITIK UND DIE SITUATION DER SAAMISCHEN SPRACHE
}

\section{EINFÜHRUNG}

Norwegen ist ein Mehrnationalitäten-, Mehrkulturen- und Mehrsprachenstaat. Die Nationalitätenvielfalt in diesem nordeuropäischen Staat bilden die auf seinem Gebiet lebenden nationalen und ethnischen Minderheiten. Im Gebiet des heutigen Norwegens leben sowohl viele einheimische als auch immigrante Minderheitengruppen.

Als Minderheiten werden Gruppen betrachtet, die in einer kleineren Anzahl in einem Staat leben und die im Vergleich zur übrigen Population über unterschiedliche ethnische, religiöse und/oder linguistische (sprachliche) Charakteristiken verfügen. Gruppen, für die diese Definition gilt, werden ethnische, religiöse oder linguistische (sprachliche) Minderbeiten genannt (Skutnabb-Kangas-Phillipson 1994:107).

Den kollektiven politischen Status betreffend, können autochthone/indigene, nationale oder immigrante Minderheiten unterschieden werden (Philipson-Rannut-Skutnabb-Kangas 1995:10).

Norwegen betrachtet als seine nationalen Minoritäten die Volksgruppen, die eine lange Verbindung zum Land haben (St.meld.nr. 15 (2000-2001)). Alle in diesem Dokument erwähnten Volksgruppen verfügen über eine mehr als hundertjährige Geschichte in Norwegen. Der norwegische Staat erkennt die Juden, die Kwenen ${ }^{1}$, die Roms, die Romanivolksgruppe ${ }^{2}$ und die Waldfinnen als seine nationalen Minderheiten an. ${ }^{3}$ Die Beibehaltung dieser kulturellen Vielfalt gehört heutzutage zu den wichtigsten Zielsetzungen Norwegens.

In diesem Aufsatz wird die saamische Minderheit, die einen speziellen Status in Norwegen einnimmt, in den Mittelpunkt der Untersuchung gestellt.

1 Eine finnische Volksgruppe in Norwegen, die aus Nordfinnland und Nordschweden gekommen ist.

2 Im betreffenden norwegischen Dokument werden beide Begriffe und Volksgruppen getrennt genannt und behandelt, obwohl die erwähnten Begriffe Gruppen gleicher Herkunft bezeichnen

3 Laut Stortingsmelding nr. 15. (2000-2001). 


\section{1. ÜBER DIE SAAMEN UND IHRE SPRACHEN}

\subsection{Die Saamen}

Die Benennung Saami ${ }^{4}$, welche in den wissenschaftlichen Texten immer häufiger vorkommt, stammt aus dem Saamischen und ist in viele Sprachen übernommen worden. Wegen historischer Ereignisse und der Norwegisierung hat die Form Lappisch/Lappe/Lappin eine negative Konnotation, deshalb werden diese Ausdrücke in der heutigen Fachliteratur vermieden.

Das Leben dieser Volksgruppe ist seit langen Zeiten an die nördlichen Gebiete des heutigen Norwegens gebunden. Deswegen verfügt sie im Vergleich zu den anderen in Norwegen lebenden Minderheiten über einen speziellen Status mit speziellen Rechten, worüber ich später ausführlicher schreibe.

Die Saamen bilden zahlenmäßig die bedeutendste Minderheit in diesem Land. Sie leben in großer Anzahl in Nordnorwegen, -schweden, -finnland und im Nordwesten von Russland, auf der Kola-Halbinsel (s. Abb. 1).

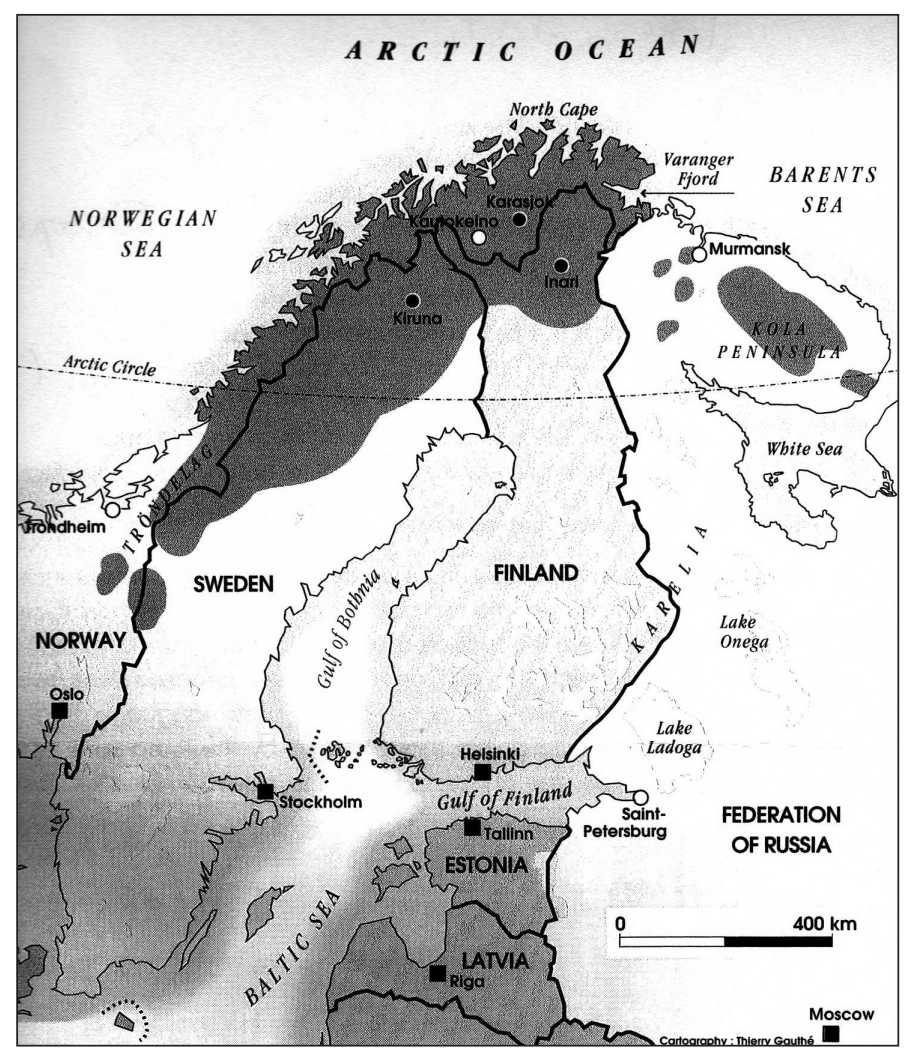

Abb. 1: Saamengebiete (The Sámi 1997:5)

Auch Sami oder Sámi geschrieben. 
Es ist sehr schwierig, ihre Gesamtzahl exakt zu bestimmen. In Norwegen erscheint in den Statistiken die Anzahl der im saamischen Verwaltungsgebiet Lebenden bzw. der Wahlberechtigten bei den Saamischen Parlamentswahlen. Heutzutage leben viele Leute saamischer Herkunft außerhalb des traditionellen saamischen Gebietes, in Finnland zum Beispiel beträgt ihr Anteil 60 \% (Sámi in Finland). In Schweden dürfen laut Gesetz keine Daten zur Ethnizität und Muttersprache gesammelt werden. Außerdem sind die Kriterien der Ethnizität sprachgebunden, aber Vermutungen nach beherrschen nur ca. 50\% der Saamen das Saamische (Spraket). Ihre Anzahl wird gewöhnlich zwischen 40000 und 60 000 geschätzt, aber nur 20 000-35 000 Personen können irgendeine Variante des Saamischen sprechen (Fodor 1999:820). Laut einiger Quellen kann die Zahl der Saamen annähernd 70000 betragen, von denen 40000 in Norwegen, 20000 in Schweden, 6000 in Finnland und 4000 in Russland leben (vgl. Wilhelm 1996:30). Nach den optimistischsten Schätzungen beträgt die gesamte saamische Population mehr als 75000 oder sogar 80000 Personen (Sámi in Finland). Laut anderer Quellen beträgt die Anzahl der Saamen zwischen 18500 und 45000 Personen, in Schweden zwischen 10000 und 25 000, in Finnland zwischen 1734 und 8000 und in Russland 2000 Personen (Greller 1996:29-30, Korhonen 1988:41).

Nach den neuesten Daten sind insgesamt ca. 70000 oder 75000 Saamen (The Sami in figures bzw. Sámi in Finnland), 23000 Saamischsprachige und ca. 40000 46000 Saamen (wenn man davon ausgeht, dass ca. 50\% der Saamen Saamisch beherrschen) in Norwegen, 9000 in Finnland (Sámi in Finland), ca. 20000 in Schweden und ca. 2000 in Russland (The Sami in figures).

Das traditionell saamische Gebiet in Norwegen ist das Gebiet von Südvaranger bis Femund's Elgå (s. Landkarte oben)

In den meisten norwegischen Gemeinden sind die Saamen heute in der Minderheit; Ausnahmen bilden nur Kautokeino mit ca. 85-90\% (Fakta om Kautokeino kommune), Karasjok mit ca. 80\% (Om Karasjok), Nesseby $(71,7 \%)$ und Tana $(54,1 \%)$ (Jávorszky 1991:28). In Finnland bildeten Saamen am Anfang der 1990-er Jahre nur in Utsjoki eine Mehrheit mit 50,1\%, heute sind 46,6\% der Bewohner Saamischsprachige (Utsjoki). In Schweden sind keine Gemeinden zu finden, wo sie eine Mehrheit bilden (Jávorszky 1991:28).

Die Saamen können in verschiedene Gruppen eingeteilt werden; die norwegischen Saamen gehören nach ihrem Siedlungsgebiet und vom sprachlichen Aspekt her zu den Nord-, Lule- und Südsaamen (vgl. Rapport om situasjonen for samisk sprk $i$ Norge 1992:1).

Laut des erwähnten Rapports werden in den drei Gruppen weitere Untergruppen unterschieden:

In den nordsaamischen Gemeinden (Kautokeino, Karasjok, Nesseby, Tana) sind die Saamen in der Mehrheit, ihre erste Sprache ist in den meisten Fällen Saamisch und das benutzen sie in den meisten Kommunikationssituationen. Die nordsaamischen Küstengebiete waren stark der Norwegisierung ausgesetzt, deshalb haben nur die älteren Generationen Saamisch als erste Sprache, und diese Sprache wird meistens nur im Familienkreis gesprochen (Jernsletten 1966:115). 


\subsection{Die saamischen Sprachen}

Die saamischen Sprachen gehören in den finnisch-ugrischen Zweig, und bilden den ostseefinnisch-saamischen Hauptzweig der uralen Sprachfamilie (Sammallahti 1998:1).

Die Sprachen existieren in vielerlei Varianten, und da zwischen einigen die Unterschiede oft groß sind, werden bestimmte Varianten als selbständige Sprachen aufgefasst. Bezüglich der Einteilung der Varianten gibt es verschiedene Vorstellungen.

Von allen Sprachgruppen verfügen sechs über eine eigene Schriftsprache, diese sind Südsaamisch, Lulesaamisch, Nordsaamisch, Inarisaamisch, Ostsaamisch (Kolttasaamisch) und Kildinsaamisch (Språket).

Über die saamischen Varietäten sind verschiedene Auffassungen bekannt. Nach einer der verbreitetsten werden die acht Dialekte oder Sprachen - Nordsaamisch, Lulesaamisch, Pitesaamisch, Umesaamisch, Südsaamisch, Inarisaamisch, Kolttasaamisch und Kolasaamisch -in drei Gruppen, d. h. Westsaamisch, Südsaamisch und Ostsaamisch eingeordnet (Fodor 1999:820). Nach einer anderen Auffassung werden Südsaamisch, Umesaamisch, Pitesaamisch, Lulesaamisch, Nordsaamisch, Inarisaamisch, Kolttasaamisch, Akkalasaaamisch, Kildinsaamisch und Tersaamisch in die Subgruppen Südsaamisch, Zentralsaamisch und Ostsaamisch eingeordnet (The Sámi 1997:22).

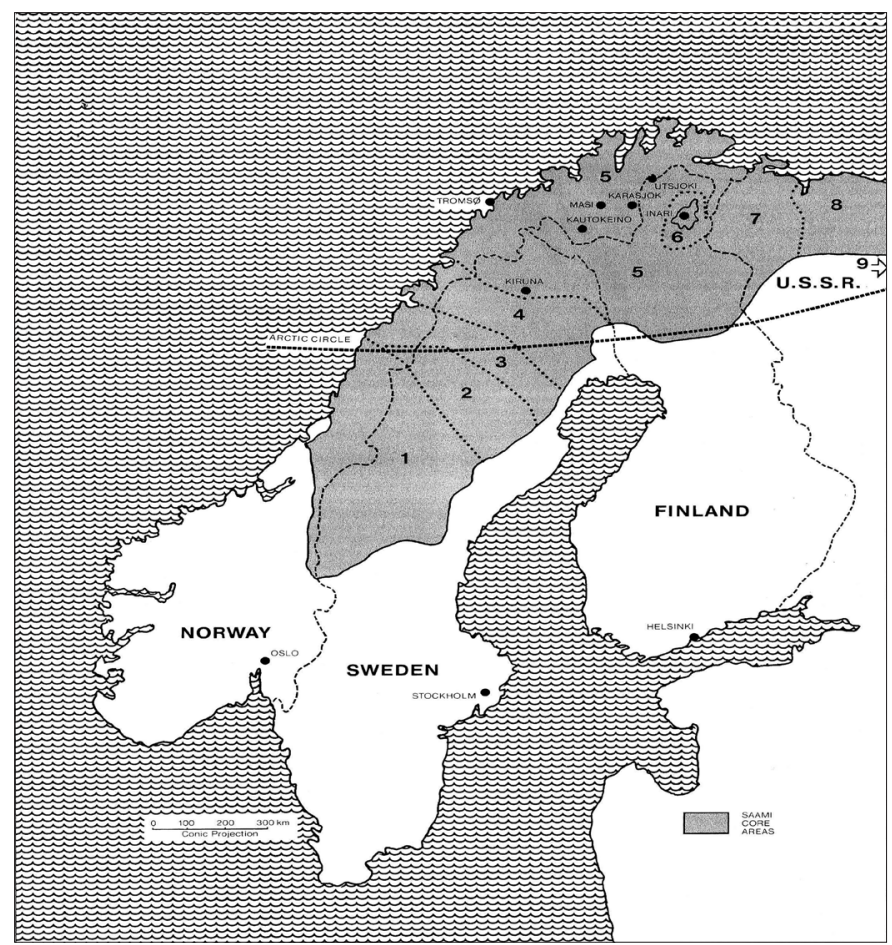

Abb. 2: Die saamischen Dialekte/Varianten (Beach 1988:3) 
Die einzelnen Sprachvarietäten bilden ein Kontinuum in den Gebieten Nordeuropas, dementsprechend unterscheiden sie sich in verschiedenem Maße. Diejenigen, die in größerer Entfernung voneinander liegen, verfügen über bedeutendere sprachliche Unterschiede. Nur diejenigen sind beiderseitig verständlich, die sich in geographischer Nachbarschaft befinden (Sami Language in Nordic Countries 2004:87).

Im Roten Buch der bedrohten Sprachen von UNESCO - in dem die Daten über die saamischen Sprachen 1993 gesammelt wurden - erscheinen auch Südsaamisch, Umesaamisch, Pitesaamisch, Lulesaamisch und Nordsaamisch. Von ihnen sind in Norwegen Ume- und Pitesaamisch als ausgestorben, Süd- und Lulesaamisch als streng bedroht, und Nordsaamisch als bedroht bezeichnet. Auch diese Tatsache konnte dazu beitragen, dass ein Revitalisierungsprozess dieser Sprachen begonnen hat (s. Huss 1999).

Dieser Aufsatz konzentriert sich auf das Nordsaamische, das sowohl in Norwegen als auch die Gesamtanzahl der Saamen betrachtet über die meisten Nutzer verfügt.

\subsection{Die saamische Volksgruppe in Norwegen}

Der früher erwähnte spezielle Status der saamischen Volksgruppe besteht darin, dass sie in diesem Staat als indigenes Volk anerkannt wurde, als die ILO-Konvention nr. 169. Übereinkommen über eingeborene und in Stämmen lebende Völker in unabbängigen Ländern (1989) von Norwegen unter den ersten Staaten im Jahre 1990 ratifiziert wurde. Dadurch sind die Saamen das einzige indigene Volk in Nordeuropa.

Laut Übereinkommen sind indigene Völker

a, in Stämmen lebende Völker in unabhängigen Ländern, die sich infolge ihrer sozialen, kulturellen und wirtschaftlichen Verhältnisse von anderen Teilen der nationalen Gemeinschaft unterscheiden und deren Stellung ganz oder teilweise durch die eigenen Bräuche oder Überlieferung oder durch Sonderrecht geregelt ist.

b, Völker in unabhängigen Ländern, die als Eingeborene gelten, weil sie von Bevölkerungsgruppen abstammen, die in dem Land oder in einem geographischen Gebiet, zu dem das Land gehört, zur Zeit der Eroberung oder Kolonisierung oder der Festlegung der gegenwärtigen Staatsgrenzen ansässig waren und die, unbeschadet ihrer Rechtsstellung, einige oder alle ihrer traditionellen sozialen, wirtschaftlichen, kulturellen und politischen Einrichtungen beibehalten.

(Teil I. Allgemeine Grundsätze, Artikel 1)

Dieses Dokument enthält die Anerkennung der wichtigsten - sowohl individuellen als auch kollektiven - Grundrechte wie das Recht auf eigenes Gebiet, auf eigene Lebensweise, Kultur, Religion und Sprache.

Die Grundlage der Saamenpolitik des Landes bilden der $§ 110$ a der Verfassung und das Saamengesetz (1987), die sich auf internationale Minderheitenschutzdokumente, wie z. B. die ILO-Konvention 169, stützen.

Laut genannter Dokumente verfügt die saamische Volksgruppe in Norwegen über ausgedehnte Rechte, sogar kulturelle Autonomie, da 1989 das Saamenparlament durch das Saamengesetz ins Leben gerufen wurde. Das war für frühere Zeiten aber nicht charakteristisch. 


\section{DIE SAAMENPOLITIK NORWEGENS IM 19. UND 20. JAHRHUNDERT. VERÄNDERUNGEN}

Bis zum 19. Jahrhundert beschäftigte man sich eigentlich nicht mit dieser Volksgruppe, die Saamen konnten ohne Probleme ihre traditionelle Lebensweise ausüben und wandern. Einen Wendepunkt bedeutete die Mitte des Jahrhunderts, die eine enorme Veränderung in der Form der Behandlung der Minderheiten mit sich brachte. $\mathrm{Zu}$ jener Zeit erwachten die nationalen Ideen und Emotionen in ganz Europa, was in Norwegen eine minderheitenfeindliche Stimmung hervorrief.

1848 brachte für die norwegischen Saamen negative Veränderungen. Dieses Jahr kann als Beginn der institutionalisierten Norwegisierung betrachtet werden, bei der die Assimilierung der Saamen das höchste Ziel war (Thuen 1980:14). 1852 kam es zum saamischen Aufstand in Kautokeino ${ }^{5}$, was die feindliche Stimmung gegenüber den Saamen noch weiter verstärkte. Dieser Aufstand kann als Protest gegen die Unterdrückung durch die norwegischen Behörden betrachtet werden (Johansen-Pettersen-Rapp 2008:19), obwohl verschiedene Theorien über dieses Ereignis erschienen sind (Brox 1997). Der Aufstand brachte eine noch schärfere Norwegisierung mit sich.

Von den 1860-er Jahren an begann die planmäßige Norwegisierung, die in zwei Phasen aufgeteilt werden kann, in eine sog. mildere und eine drastischere.

Die erste Epoche, die „weiche“ Phase der Liquidierung der saamischen Minderheit dauerte bis 1880. In diesem Zeitraum wurden spezielle Kindergärten geschaffen, damit die Kleinkinder so früh wie möglich mit dem Norwegischlernen beginnen. Neben Mitteln der Erziehung und des Unterrichts spielte die Ansiedlung von Norwegern eine wichtige Rolle beim Norwegisierungsprozess.

Während der „harten“ Phase wurde eine richtig drastische Norwegisierung durchgeführt: Maßnahmen wurden getroffen, die die Assimilation der Saamen (und anderer Minderheitenvolksgruppen) förderten. 1880 wurde ein neuer Wegweiser, eine methodische Instruktion für die Schulen eingeführt, die vorschrieb, dass alle Kinder saamischer und finnischer Herkunft Norwegisch schreiben, lesen und sprechen lernen sollen (Jávorszky 1991:36). Parallel dazu wurde aus deren Text die Vorschrift des Lernens/Unterrichts der Muttersprache gestrichen (Jávorszky 1991:59). Für diese Zielsetzung wurden die (bis dahin benutzten) zweisprachigen Lehrbücher eingezogen, der Finnisch- und Saamischunterricht eingestellt, außerdem wurde die Benutzung der Muttersprache in den Schulen während und außerhalb der Stunden verboten (Lund 2003:20). In Finnmark wurde das Schulsystem im Laufe einiger Jahre völlig umstrukturiert (vgl. Dahl 1957).

Die spätere Wexelsen-Verordnung (1898), die die gültigen Dokumente verschärfte, stand bis in die Jahre nach dem Zweiten Weltkrieg in Kraft.

\footnotetext{
Eine Gruppe von Saamen erschlug einen Kaufmann, bei dem sie gegen ihre Produkte Branntwein tauschte, sie erschlug auch einen Polizisten und verprügelte einen Pfarrer. Insgesamt wurden 33 Saamen verurteilt, davon fünf Teilnehmer zum Tode, acht zu lebenslanger Haft (Johansen-Pettersen-Rapp 2008:19).
} 
Da mit den erwähnten Maßnahmen die angestrebten Ergebnisse nicht erreicht werden konnten, nahm auch die Kirche aktiv an den Norwegisierungstätigkeiten teil, vor allem durch Errichtung von Internaten und Volkshochschulen (Jávorszky 1991:60). ${ }^{6}$

Auf dem Gebiet des Unterrichts griff der norwegische Staat gewaltsam ins Leben seiner Minderheiten ein, indem er ihnen keine Möglichkeit ließ, ihre Muttersprache zu erlernen und einzuüben oder zu erhalten. Eine Folge dieser groben Norwegisierungspolitik durch den Unterricht ist die, dass die Zahl der Saamen, die in ihrer Muttersprache schreiben und lesen können, relativ niedrig ist. Laut einer Untersuchung von $2000^{7}$ können 31\% der Saamen nicht Saamisch lesen und $46 \%$ können nicht Saamisch schreiben.

Außerhalb des Unterrichts war die Ansiedlung von Norwegern eine bekannte Methode im Interesse der Norwegisierung der Saamen, wodurch sich der Prozentsatz der saamischen und norwegischen Bevölkerung veränderte. Von 1870 an kamen im Verlaufe von 60 Jahren 50-60000 Norweger in die typischen Saamengebiete und dadurch sank die Zahl der Saamen bedeutend. Während Anfang des 19. Jahrhunderts die Zahl der Saamen dreimal so hoch war wie die der Norweger, verdoppelte sich bis zur Jahrhundertwende die Zahl der Norweger im Vergleich zu den Saamen.

Das Bodengesetz von 1902 passte gut zu diesen Maßnahmen, das den Bodenkauf nur denjenigen erlaubte, die einen norwegischen Namen hatten und Norwegisch sprechen und schreiben konnten (Tófalvi 2000:65).

Nach dem Zweiten Weltkrieg änderte der norwegische Staat seine Minderheitenpolitik, hörte mit seiner drastischen Norwegisierung auf, unterstützte sogar die saamische Kultur und Sprache (Stenersen-Libæk 2003:163).

Die Verbesserung des Verhältnisses zwischen den Norwegern und den Saamen ist zum Teil dem Weltkrieg und den Kriegstaten der Deutschen zu verdanken. Weiterhin hängen diese Veränderungen auch mit der erstarkenden Organisierung der Saamen ab Mitte des 20-en Jahrhunderts zusammen.

Lange Zeit organisierten sie sich nicht, hatten keine eigenen Organisationen, in denen sie für ihre Rechte kämpfen konnten. Das konnte mehrere Gründe haben: Diese Volksgruppe war jahrzehntelang Opfer von Diskriminierung und Unterdrückung, darüber hinaus lebt sie in vier Staaten verstreut und hat keinen eigenen Staat. Als Folge der Diskriminierung brachen viele Saamen mit ihrer Herkunft, verließen ihren Wohnort und wurden „Norweger“ (Stenersen-Libæk 2003:163).

Die Organisierung der Saamen konnte Ende der 1940-er Jahre beginnen; von da an entstanden mehrere bedeutende und weniger bedeutende Gruppen bzw. Organisationen, sogar eine Saamenvereinigung von Saamen in Finnland, Schweden und Norwegen (Stenersen-Libæk 2003:163-165).

Eine entscheidende Rolle spielte bei der Organisierung der Saamen und ihrer Stärkung als Gemeinschaft der Naturschutz-Widerstand gegen den Kraftwerk-

\footnotetext{
6 Es gab dagegen Geistliche und Lehrer, die Interesse für die saamischen Sprachen zeigten und sie auch unterstützten.

7 SEG 2000. Rapport. Undersøkelse om bruken av samisk språk
} 
bau in Altafjord, die sog. Alta-Sache. In den 1970-er Jahren kam es zum Hungerstreik und mehreren Demonstrationen, die die Aufmerksamkeit auf die Saamen und die Saamenfrage richteten. Zeitlich knüpften sich daran die internationalen Bewegungen auf dem Gebiet der Minderheitenrechte; damals organisierten sich nämlich die Urvölker (Bjørklund 2003:3).

Als wichtiges Ergebnis zählt die Bewilligung der Gründung des Saamischen Parlaments ${ }^{8}$ im Jahre 1989 (Stenersen-Libæk 2003:164). Dieser Schritt hatte Vorereignisse: die Ernennung einer Kommission für saamische Angelegenheiten. Als Ergebnis der Arbeit dieser Kommission entstand das Saamengesetz ${ }^{9}$, welches die Grundlage des Saamischen Parlaments bildete.

Der norwegische Staat hat die grundliegende Zielsetzung, seinen nationalen und internationalen Rechtsverbindlichkeiten nachzukommen. Diese Ziele bilden die Saamenpolitik Norwegens. Die Regierung hält die (sprachlichen) Rechte der Saamen für vorrangig, was sich in den ministerialen Pressemeldungen, in Konferenzvorträgen und in den konkreten Maßnahmen zeigt.

Dass die nördlichen Länder die Saamenfrage ernst nehmen, zeigt der Zusammenhalt von Norwegen, Schweden und Finnland. Die für die saamischen Angelegenheiten zuständigen Minister und die Präsidenten der saamischen Parlamente der genannten Staaten beschlossen 2001 eine Saamenkonvention ${ }^{10}$, die die Definition und den Status der Saamen, den Rahmen der Zusammenarbeit zwischen den Staaten und den samischen Parlamenten, die Regelung von Sprache, Kultur, Unterricht, Forschung, Gesundheit sowie Kinder und Jugendliche betreffende Fragen enthalten soll (Samepolitikk).

In den offiziellen Dokumenten ist die Absicht Norwegens offensichtlich, den Gebrauch des Saamischen in immer mehr Lebensbereichen anzuregen. Diesem Zweck dienen auch die Eröffnung der saamischsprachigen Internetseiten der Ministerien und die finanzielle Unterstützung von Projekten, die die Stärkung der saamischen Sprache bezwecken.

\section{DIE SAAMENPOLITIK NORWEGENS HEUTE}

Längere Zeit arbeitete die Abteilung für Saamen- und Minderheitenpolitik innerhalb des Arbeitsministeriums, vom 1. Januar 2010 an gehört sie zum Ministerium für Neuerungen, Administration und Kirchen. Diese Abteilung ist für die Ausarbeitung und Koordinierung der zentralen Saamen- und Minderheitenpolitik zuständig. Sie vertritt das Saamische Parlament im norwegischen Parlament und vor der Regierung, koordiniert aber auch die Kontakte und den Dialog zwischen den Ministerien. Außerdem beschäftigt sie sich mit der grundsätzlichen Förderung und Projektunterstützung von Minderheiten und leitet die Zentren für einheimische Volksgruppen und Rentierhaltung (Fornyings-, Administrasjons- og Kirkedepartementet).

\footnotetext{
Sameting

Samelov

Nordisk samekonvensjon
} 
Die norwegische Regierung fördert auch finanziell die in Norwegen lebenden nationalen Minderheiten. Die Minderheiten können in zweierlei Formen Fördermittel bekommen: Ihre Vertretungsorgane erhalten eine sog. Grundförderung, daneben können Projekte gefördert werden, wenn sie sich bei Minderheitenfragen mit der Diskriminierung befassen.

\subsection{Saamisch im Unterricht}

\subsubsection{Gesetz über die Grundschule und die Mittelschule ${ }^{11}$}

Laut erster und allgemeinster Verordnung dieses Gesetzes aus dem Jahre 1998 haben alle Schulpflichtigen das Recht auf Unterricht des Saamischen und auf Saamisch im saamischen Distrikt ${ }^{12}$. Außerhalb dieses (im Saamengesetz konkret beschriebenen) Gebietes gelten andere Regeln, aber auch dort haben die Schüler Recht auf Unterricht des Saamischen oder auf Saamisch: In einer Gemeinde sind mindestens zehn Personen zur Einführung des Unterrichts nötig.

Auch in den Mittelschulen haben die Schüler ein Recht auf Saamischunterricht. Wenn das mit der Lehrkraft der gegebenen Schule nicht zu lösen ist, können seine Formen vom Ministerium bestimmt werden. Die Fylkeskommune ${ }^{13}$ kann auch Sprachkurse oder Kurse mit saamischem Inhalt anbieten.

Die für die Lehrpläne für den Saamischunterricht in den Grund- und Mittelschulen und für die speziellen saamischen Fächer in den Mittelschulen geltenden Vorschriften stammen vom Saamischen Parlament, unter Beachtung der Verordnungen des Ministeriums. In anderen Fällen ist die Regelung der Lehrpläne in den Händen des Ministeriums.

\subsubsection{Saamischsprachiger Unterricht}

Von Saamischunterricht und Unterricht auf Saamisch in Norwegen kann man seit der zweiten Hälfte des 20. Jahrhunderts sprechen. Dessen Wurzeln gehen auf frühere Zeiten zurück: Schon im 17. Jahrhundert wurden die ersten Schulen speziell für saamische Kinder geschaffen (Lund 2003:10). Das nächste Jahrhundert brachte aber eine radikale Änderung, als sich die nationalen Ideen stärkten (Myrvoll 1999:29). Damals hatten die saamischen Kinder keine Möglichkeit ihre Muttersprache zu lernen, und der Gebrauch ihrer Muttersprache war sogar außerhalb der Stunden strengstens verboten (Myrvoll 1999:29). Nach dem Zweiten Weltkrieg begann sich diese Situation zu ändern und Saamischunterricht bzw. der Unterricht auf Saamisch wurde wieder ermöglicht (St.meld. $n r .15,4.5)$.

Heutzutage ist im zentralen saamischen Gebiet - d.h. in Norwegen in Kautokeino, Karasjok, Tana und Nesseby - ein vollständiger Unterricht auf Saamisch möglich. Seit 1974 erscheinen in den Lehrplänen in einem selbstständigen Kapitel das Recht der dort lebenden Kinder auf Saamischunterricht oder auf den

11 Lov om grunnskolen og den vidaregåande opploeringa

12 Es wird auch saamischsprachiges Verwaltungsgebiet genannt, das zur Zeit aus den Gemeinden Karasjok, Kautokeino, Nesseby, Porsanger, Tana, Kåfjord, Tysfjord, Snåsa und Lavangen besteht.

13 Fylke ist eine größere Einheit als die Gemeinde und eine kleinere als die Region 
Unterricht auf Saamisch und die Zielsetzungen für den Saamischunterricht. Von der siebten Klasse an können die Schüler entscheiden, ob sie Saamisch lernen wollen. Wenn jemand Saamisch wählt, wird er vom Erlernen einer Variante des Norwegischen ${ }^{14}$ in den folgenden zwei Jahren befreit.

Im Lehrplan aus dem Jahre 1987 werden die Fächer Saamisch als erste Sprache und Saamisch als zweite Sprache beschrieben. Saamisch als zweite Sprache kann man $\mathrm{ab}$ zweiter oder dritter Klasse lernen; in diesem Fall geht es um keinen muttersprachlichen Unterricht, sondern er ist dem Fremdsprachenunterricht ähnlich.

Für heute wurden parallel zu den Nationallehrplänen selbstständige, gleichwertige Lehrpläne für die saamischen Gebiete (und für den Saamischunterricht) ${ }^{15}$ entwickelt. Neu in diesem Lehrplan ist die Betonung des Lernens des Saamischen und Norwegischen, damit die Entwicklung der Zweisprachigkeit der Schüler verwirklicht werden kann.

Unterricht auf Saamisch erfolgt in mehreren Grundschulen, Mittelschulen mit saamischsprachigem Unterricht gibt es in Karasjok und Kautokeino. Außerdem wird Saamisch in vielen Mittelschulen in Nordnorwegen unterrichtet (St. meld. nr. 34, 5.2).

In einigen Schulen sind die nötigen Bedingungen dazu nicht vorhanden. In den meisten Fällen werden Lehrer-, eventuell Lehrbuchmangel erwähnt (Jávorszky 1991:47). Mit der Zeit scheint dieses Problem - vor allem durch die Lehrerausbildung der Saamischen Hochschule und die immer größere Auswahl von saamischsprachigen Lehrbüchern - gelöst zu sein.

Studieren auf Saamisch kann man an einer Hochschule, an der früheren Saamischen Hochschule. Diese Hochschule ist heute für die Lehrerausbildung für Saamisch zuständig. An anderen Institutionen des Landes werden Quoten für saamische Bewerber - die im saamischen Register stehen - bestimmt (St.meld. $n$ r. 34, 5.4).

Studien und Forschungen von saamischen Themen sind an mehreren Universitäten und Hochschulen möglich, wie z. B. in Bodø, Finmark und Tromsø. An der Universität Tromsø besteht seit 1990 sogar ein selbstständiges Zentrum für Saamische Studien.

An der Universität Oslo hatten Studien der saamischen Sprache Tradition, aber ab den 1990-er Jahren gibt es keine solchen Studien mehr (St.meld. nr. 34, 4.6.5).

\subsection{Ortsnamengesetz ${ }^{16}$}

Dieses Gesetz regelt den Gebrauch von Ortsnamen mehrsprachiger Gebiete. Dementsprechend können saamische Ortsnamen öffentlich auf Ortsnamentafeln, Landkarten und Stadtplänen, in Ortsnamenregistern gebraucht werden, eventuell mit der norwegischen Variante zusammen $(\S 3, \S 3-2)$. Die Reihenfolge der Formen ist bestimmt: im saamischen Verwaltungsgebiet erscheint zuerst der sa-

\footnotetext{
14 Bokmål oder nynorsk, beide müssen norwegische Schüler in der Schule lernen (die eine als Haupt-, die andere als Nebensprache)

15 Kunnskapsloftet-Samisk

16 Stedsnavnlov
} 
amische Name, dann der norwegische (eventuell noch der finnische). In anderen Fällen soll die norwegische Variante vor der saamischen oder/und der finnischen stehen.

\subsection{Aktuelle Dokumente über die Saamenpolitik}

\subsubsection{Parlamentsmeldungen}

Stortingsmelding $n r .33$ (2001-2002) beinhaltet die Regelungen über die Saamenpolitik, und betrachtet als Ausgangspunkt das europäische Prinzip, wonach die sprachliche Vielfalt ein Land bereichert. Als Ziel erscheint die Entwicklung der Zweisprachigkeit. Dazu sieht die Regierung eine höhere Förderung vor, die durch das Saamische Parlament verteilt wird.

In der Bewahrung und Verbreitung der saamischen Sprache - was ein Grundziel der norwegischen Politik ist - spielen die Stipendien für Schüler, die in einer nicht-saamischen Mittelschule Saamisch lernen, eine wichtige Rolle.

Stortingsmelding $n r .48$ (2002-2003) plant langfristig, darin werden die Zielsetzungen, Richtlinien der norwegischen Kulturpolitik bis 2014 bestimmt. Dieses Dokument erwähnt die Bewahrung und Entwicklung der saamischen Sprache als eine unentbehrliche Aufgabe, deshalb möchte das zuständige Ministerium mit dem Saamischen Parlament zusammenarbeiten. Am wichtigsten finde ich, dass die Übernahme der Verantwortung für die saamische Sprache einen wichtigen Teil der ganzen norwegischen Sprachen- und Kulturpolitik bildet.

Seit 2003 gehört die Bewahrung und Entwicklung der saamischen Sprache zu den Aufgaben des Saamischen Parlaments.

Eine der neuesten Regierungsmaßnahmen ist ein Handlungsplan für die saamische Sprache aus dem Jahre 2009, der sich auf die Entwicklung des Unterrichts des Saamischen bzw. auf Saamisch im Kindergarten, in den Schulen und in der Erwachsenenbildung, auf die Verbreitung dieser Sprache in den öffentlichen Institutionen und in den Medien konzentriert. ${ }^{17}$

\section{SAAMISCHE ORGANISATIONEN, INSTITUTIONEN, KULTURELLE EINRICHTUNGEN UND IHRE ROLLE BEI DER BEWAHRUNG UND PFLEGE DER SAAMISCHEN SPRACHE}

In der letzten Zeit wurden immer mehr Organisationen, politische Parteien gegründet, die die saamischen Interessen am besten vertreten können.

Das früher schon erwähnte Saamische Parlament ist keine spezielle norwegische Erscheinung, solche findet man nicht nur in Norwegen, sondern auch in Schweden und Finnland. Es ist die bedeutendste Institution der saamischen Volksgruppe, es ist ihr politisches Vertretungsorgan und besteht aus direkt gewählten Abgeordneten (Samelov §2-3). Es ist selbstständig, in seine Entscheidungen kann die norwegische Regierung nicht eingreifen. Es kann Vorschläge ma-

17 Handlingsplan for samiske språk. 
chen, eine von der Regierung unabhängige Politik führen in Angelegenheiten, die die saamische Bevölkerung betreffen. Zu seinem Kompetenzbereich gehören die saamischen kulturellen Institutionen wie das Theater, Sammlungen, Festivals, Kunststipendien und saamische Bücherbusse.

Über die Verteilung von finanziellen Mitteln kann das Saamische Parlament nach der Entscheidung des norwegischen Parlaments über das Budget beschließen. Diese Fördergelder kommen aus mehreren Ministerien zum Saamischen Parlament (Finansdepartementet. Statsbudsjettet).

Das Saamische Parlament verfügt auch über eine eigene Bibliothek ${ }^{18}$, die die größte saamischsprachige Sammlung Norwegens ist.

Mit dem Saamengesetz kam das unter der Aufsicht des Saamischen Parlaments stehende Verwaltungsorgan, der Saamische Sprachrat zustande. Aufgaben des Rates sind die Bewahrung und Entwicklung der saamischen Sprache, so wie auch Dolmetscherdienst, Terminologieentwicklung und Fragen der Zweisprachigkeit (Myrvoll 1999:75).

Mit dem Saamischen Rat entstand 1956 eine von der Regierung unabhängige kulturpolitische und politische Institution, die für alle saamischen Gruppen zuständig ist. Seine Bedeutung ist die Zusammenarbeit der Saamen, von samischen Institutionen jenseits der Grenzen, in den Staaten, wo Saamen leben. Ziel dieser Maßnahme war es, die Saamen wirklich als ein einheitliches Volk zu anerkennen (Samerådet Organisasjon).

Das Saamische Institut begann 1973 seine Arbeit, die mit der saamischen Sprache zusammenhängt. Seine Forschungsergebnisse über die Struktur und den Gebrauch des Saamischen werden gewöhnlich in der wissenschaftlichen Reihe DIEĐUT veröffentlicht. Heute erscheint sie nicht mehr selbstständig, sondern im Rahmen der Saamischen Hochschule.

Die Saamische Hochschule wurde 1989 in Kautokeino gegründet, eine Institution mit gesamtsaamischem Profil; d. h. sie ist offen für Studenten, Professoren und Forscher aus Norwegen, Schweden, Finnland und Russland. Die Hauptbildungsgebiete knüpfen an die saamische Lebensweise und Kultur, so wie an Sprache, Pädagogik/Gesellschaft, Rentierhaltung/Natur und Duodji ${ }^{19}$. Auf die saamische und einheimische Perspektive wird Wert gelegt. Die Unterrichtssprache ist Saamisch (Nordsaamisch), aber es wird auch in der Administration und in der Kommunikation zwischen Professoren und Studenten gebraucht.

In der Bekanntmachung der saamischen Sprache und Kultur spielen saamische Jugendliche, Studenten der Saamischen Hochschule eine bedeutende Rolle. Jedes Jahr werden drei sog. guides aus den drei Hauptgruppen der Saamen (Nord-, Lule- und Südsaamisch) gewählt. Als guides suchen sie Schulen auf und informieren die norwegischen Schüler über die saamische Kultur. Durch diese Tätigkeit kann man die saamische Kultur, die saamischen Leute besser kennen lernen, und das kann zur Herausformung einer positiven Einstellung zu den Saamen beitragen.

\footnotetext{
18 Samisk spesialbibliotek

19 Saamisches Handwerk. Es werden überwiegend Gebrauchsgegenstände aus Naturstoffen, meistens aus Renknochen und -Haar hergestellt.
} 
Heutzutage existieren mehrere saamische Sprachzentren, einige davon sind Várdobáiki, Ája, Árran, Isak Saba, deren Hauptfunktion die Pflege, Entwicklung und Verbreitung der saamischen Sprache und Kultur ist. Diese Institutionen sind mehrfunktionelle Institutionen, d. h. sie organisieren Ausstellungen mit saamischem Inhalt, veranstalten kulturelle Programme und Vorstellungen. Außerdem spielen sie in der Sprachbewahrung eine hervorragende Rolle: in allen Sprachzentren werden regelmäßig Saamischkurse auf verschiedenen Stufen organisiert. Das Zentrum Árran für Lulesamisch bietet z. B. Videokonferenzkurse für Grund- und Mittelschulen und führt auch einen Kindergarten.

Vor ein paar Jahren wurde ein saamisches Kulturzentrum in Oslo, das Samisk hus von mehreren Organisationen und Parteien gegründet.

Das Saamische Theater, welches als vierte nationale Bühne Norwegens bekannt ist, besteht seit 1981 in Kautokeino. Vor kurzem wurde es aus dem Budget des Staates ins Budget des saamischen Parlaments verlegt.

Das Saamische Archiv in Kautokeino hat eine sehr wichtige Aufgabe bei der Sammlung, Bewahrung und Vorstellung von Quellen der saamischen Geschichte.

In unseren Tagen sind mehrere saamischsprachige und andere Zeitungen, Zeitschriften mit saamischem Inhalt auf dem Markt. Die auf Nordsaamisch überwiegen. Die bekanntesten in Norwegen erscheinenden saamischsprachigen sind Nuorttanaste (die älteste), Min Áigi, Ǎšsu, die vom Saamischen Frauenforum herausgegebene Gába, das Magazin $S$ für Jugendliche, auf Norwegisch erscheint Ságat. Weitere Zeitungen und Magazine werden in Finnland und Schweden herausgegeben: in Finnland Ođđa Sápmelaš überwiegend auf Nordsaamisch, Anarâs auf Inarisaamisch, in Schweden Samefolket auf Schwedisch und Nordsaamisch, Sáminuorra für Jugendliche auf Schwedisch, gelegentlich mit einer Seite auf Nordsaamisch. Weitere bekannte und beliebte Zeitungen sind Sápmi, Ávvir, und SFFNettopp nu im Internet.

Saamischsprachige Radiosendungen haben alle drei skandinavische Länder, in Norwegen waren sie zum ersten Mal $1948 \mathrm{zu}$ hören (Huss 1999:159). Heute ist NRK Sámi Radio das bekanteste, das auch im Internet erreichbar ist.

Im Fernsehen, im norwegischen Kanal NRK - und auch im Internet - ist jeden Tag eine nordische Gesamtproduktion, das saamischsprachige Nachrichtenprogramm OĐĐASAT TV zu sehen.

\section{NORDISCHE ZUSAMMENARBEIT}

Die Saamen gestalteten ihre Zusammenarbeit auf internationaler Stufe ab Anfang der 1950-er Jahre. Die nordische Saamenkonferenz im Jahre 1953 in Schweden begründete diese Zusammenarbeit, die durch die Gründung des Saamischen Rates institutionalisiert wurde. Der Rat bestimmt gemeinsame Zielsetzungen, formt gemeinsame Politik und entscheidet darüber.

Ein sehr gutes Beispiel für die Zusammenwirkung in saamischen Fragen ist die Zusammenarbeit der norwegischen, schwedischen und finnischen Regierung. Ihr größtes Ziel ist die Ausarbeitung einer nordischen Saamenkonvention, deren 
Entwurf zur Zeit in den drei nordischen Ländern unter Diskussion steht (Forhandlingene om nordisk samekonvensjon er igang).

\section{ZUSAMMENFASSUNG}

Man kann sehen, dass die norwegische Saamenpolitik in den letzten Jahrzehnten eine enorme Veränderung erlebte. Im 19. und 20. Jahrhundert wurden die Minderheiten - am meisten die Saamen und die Finnen - auf dem Gebiet des heutigen Norwegens stark unterdrückt. Das zeigte sich am intensivsten an der Beschränkung des Gebrauchs ihrer Muttersprache.

Heute wird die saamische Minderheit in diesem Staat als indigenes Volk anerkannt und dementsprechend verfügt sie über ausgedehnte Rechte, sprachliche Rechte inbegriffen.

Das Saamische wird - innerhalb des saamischen Verwaltungsgebietes - dem Norwegischen gleichgestellt. Das bedeutet, dass es auch im offiziellen Bereich, in der Verwaltung, in Büros, in Krankenhäusern gebraucht werden kann. Die Kinder und Jugendlichen können saamischsprachigen Unterricht wählen oder Saamisch lernen.

In unseren Tagen sind die rechtlich-institutionellen Bedingungen für die Bewahrung der saamischen Sprache und Kultur gegeben, deshalb scheint die Einstellung der saamischen Volksgruppe der bedeutendste Faktor bei der Bewahrung und Entwicklung der saamischen Sprache und Kultur zu sein. Diese Aussage gilt in größtem Maße innerhalb des saamischen administrativen Gebietes.

Trotz des besonderen Status und ausgedehnter Rechte sind die Saamen nicht vollständig zufrieden. Einige Mitarbeiter eines saamischen Zentrums, die ich im Rahmen meiner Untersuchungen befragt habe, beurteilen die norwegische Minderheiten- und Minderheitensprachenpolitik unterschiedlich. Die Meinungen über die Minderheitenpolitik sind verschieden, von sehr gut bis mittelmäßig, über die Minderheitensprachenpolitik von ausreichend bis eher nicht ausreichend. $\mathrm{Zu}$ den wichtigsten minderheitensprachpolitischen Einrichtungen und Tätigkeiten werden die saamischsprachigen Kindergärten, die Saamischen Zentren und der Saamischunterricht und der Unterricht auf Saamisch in den Schulen gezählt. Außerdem finden sie die saamischsprachigen Zeitungen, Fernseh- und Radioprogramme besonders bedeutend. Als Mängel der Minderheitensprachenpolitik Norwegens werden die fehlenden Schritte für die Verbesserung in der Einstellung der Norweger zur saamischen Sprache erwähnt. Der Staat sollte mehr finanzielle Unterstützung für saamischsprachige Bücher, Magazine, Filme, und Homepages geben. Überdies sollten auch die Bedingungen der saamischsprachigen Kommunikation verbessert und entwikkelt werden. 


\section{LITERATUR}

ANDERSEN, Svanhild/Johan STRÖMGREN (2007) Evaluering av samelovens språkregler/Sámelága giellanjuolggadusaid evalueren. Utredning/Čielggadeapmi.

BEACH, Hugh (ed.) (1988) The Saami of Lapland. The Minority Rights Group, Report no. 55.

BJØRKLUND, Ivar (2003) Sápmi. Eine Nation entsteht. Tromsø: Tromsø Museum, Universität in Troms $\emptyset$.

BROX, Johan (1997) Kautokeino-opprøret: Kautokeino 1952. In: Dag og Tid nr. 16. http://www.dagogtid.no/arkiv/1997/16/kauto1.html (Zugriff: 28.11.2010)

Fakta om Kautokeino kommune.

http://www.kautokeino.kommune.no/finnmark/kautokeino/kautokeino. nsf/id/F195C4E3B5CB172241256C5500485580?OpenDocument (Zugriff: 15.09.2010)

Finansdepartementet. Statsbudsjettet.

http://www.statsbudsjettet.dep.no (Zugriff: 30.11.2010)

FODOR, István (ed.) (1999) A világ nyelvei. Budapest: Akadémiai Kiadó.

Forhandlingene om nordisk samekonvensjon er igang.

http://www.samediggi.no/artikkel.aspx?MId1=3296\&MId2=3296\&MId3=3 296\&AId=

3987\&Back=1 (Zugriff: 28.11.2010)

Fornyings-, Administrasjons- og Kirkedepartementet.

http://www.regjeringen.no/nb/dep/aid/dep/org/avdelinger/SAMI.html?id $=1453$ (Zugriff: 07.12.2010)

GRELLER, Wolfgang (1996) The Sámi language(s) Maintenance and Intellectualisation. In: Bull, Tove: Current Issues in Language Planning, Vol. 3. Issue 1, 2002. S. 28-39.

\section{Historien.}

http://sametinget.no/artikkel.aspx?AId=54\&back=1\&MId1=11\&MId2=122 (Zugriff: 10.09.2008)

HUSS, Leena (1999) Reversing Language Shift in the Far North. Uppsala University: Studia Uralica Upsaliensia 31.

JÁVORSZKY Béla (1991) Észak-Európa kisebbségei. Budapest: Magvető Kiadó.

JERNSLETTEN, Nils (1966) Sami language communities and the conflict between Sami and Norwegian. In: Haugen, Einar: Language Conflict and Language Planning. The Case of modern Norwegian. Cambridge, Massachussetts: Harvard University Press. S. 115-133.

JOHANSEN, Elisabeth/John Roald PETTERSEN, Ole Magnus RAPP (2008) Samene-Kultur og historie. Orkana: Stamsund.

Rapport om situasjonen for samisk språk $i$ Norge $i$ 1992. Kautokeino: Samisk Språkråd. 
LUND, Svein (2003) Samisk skole eller Norsk Standard? Reformene i det norske skoleverket og samisk opplæring. Karasjok: Davvi Girji.

MYRVOLL, Marit (1999) Samene -ett folk i fire land: Samisk innhold i læreplanverket -en ressursbok for lærere in grunnskolen. Samisk Utdanningsråd.

Om Karasjok.

http://www.karasjok.kommune.no/document.aspx?uid=60\&title=Fakta

(Zugriff: 10.09.2010)

Om Sametinget.

http://www.sametinget.no/artikkel.aspx?MId1=3377\&AId=3645\&back=1\& MId2=3483 (Zugriff: 05.06.2010)

PHILLIPSON, Robert/Mart RANNUT, Tove SKUTNABB-KANGAS (1995) Introduction.

Samerådet. Organisasjon.

http://www.saamicouncil.net/?deptid=1203 (Zugriff: 05.06.2010)

Sámi in Finland.

http://www.samediggi.fi/index2.php?option=com_content\&task=view\&id $=71 \&$ pop $=\ldots$ (Zugriff: 22.11 .2010$)$

Sami language in Nordic countries (2004) Diedut nr. 4/2004.

SAMMALLAHTI, Pekka (1998): The Saami Languages. An Introduction. Kárášjohka/Karasjok: Davvi Girji.

SEG 2000. Rapport. Undersøkelse om broken av samisk språk.

SKUTNABB-KANGAS, Tove/Robert PHILLIPSON (1995) Linguistic Human Rights: Overcoming Linguistic Discrimination. Berlin; New York: Mouton de Gruyter.

Språket. (John-Marcus Kuhunnen)

http://sametinget.no/artikkel.aspx?AId=56\&back=1\&MId1=11\&MId2=123 (Zugriff: 16.08.2010)

STENERSEN, Øivind/Ivar LIBAEK (2003) Die Geschichte Norwegens von der Eiszeit bis heute. Lysaker: Dinamo Forlag.

The Sami in figures.

http://www.eng.samer.se/servlet/GetDoc?meta_id=1536 (Zugriff: 11.10.2010)

THUEN, Trond (ed.) (1980) Samene - urbefolkning og minoritet. Troms $\varnothing-O s l o-$ Bergen: Universitetsforlaget.

TÓFALVI, Zoltán (2000) Északi változatok. Marosvásárhely: Mentor Kiadó.

UNESCO Red Book on Endangered Languages: Europe.

http://www.helsinki.fi/ tasalmin/europe_report.html (herunterladen 28.11.2010)

Übereinkommen 169 über eingeborene und in Stämmen lebende Völker, 1989. http:// www.gfbv.it/3dossier/diritto/ilo169-conv-dt.html (Zugriff: 12.08.2010)

WILHELM, Gábor (1996) Kultúra és egyebek: a lappok esete Észak-Európában. In: Regio 1996/1. S. 26-43. 


\section{POVZETEK}

\section{Spremembe v norveški politiki obravnave Laponcev in položaj laponskega jezika}

$\mathrm{V}$ članku avtorica predstavlja spremembe norveške politike do laponske manjšine. Laponska manšina je najštevilčnejša manjšina na Norveškem, ima poseben položaj in ji država zagotavlja posebne (zlasti jezikovne) pravice. Pot do sedanjih pravic je bila težka, saj laponska manjšina $v$ preteklosti ni bila $v$ enakovrednem položaju.

Pomembno obdobje za laponsko manjšino je nastopilo v drugi polovici 50. let prejšnjega stoletja. Laponske organizacije in insitucije so bile ustanovljene druga za drugo, prav tako so se pojavili zakoni, ki so uravnavali pravice manjšine. Najpomembnejši za to so bili ideološki trendi ter prebujanje in organiziranje laponske manjšine.

Sedanji sistem je lahko zgled za druge države. Preden je bil vzpostavljen, je prešel obdobja pomembnih sprememb; najpomembnejše avtorica predstavlja v članku.

Ključne besede: laponska manjšina na Norveškem, pravice, zakoni

\section{ABSTRACT}

\section{Changes in the Norwegian Samipolicy and the Situation of the Sami Language}

In this article I would like to present the changes in the policy of Norway concerning its indigenous minority, the Sami. This minority group is the most numerous minority group in Norway, it has a special status in this country and the state provides special (language) rights for it. The way to the present situation was complicated, from the hard Norwegization to a democratic Sami policy.

The second half of the 1950s brought a great breakthrough, and the Sami organizations, institutions and laws concerning the Sami were established one after the other. The most important reasons of this change were the international ideological trends, the awakening among the Sami and the organization of them.

The present system can be exemplary for other countries but it passed through important changes until it achieved its present form, and here the most important periods and changes will be presented.

Key words: Norwegian Samipolicy, Sami minority, rights, laws 